\title{
Transvaginal mesh failure: lessons for regulation of implantable devices
}

Carl Heneghan, Jeffrey K Aronson, Ben Goldacre, Kamal R Mahtani, Annette Plüddemann, Igho Onakpoya

Author affiliations

${ }^{1}$ Centre for Evidence-Based Medicine, Nuffield Department of Primary Care Health Sciences, University of Oxford, Oxford OX2 6GG, UK

Correspondence to: C Heneghan carl.heneghan@phc.ox.ac.uk 
Concern is increasing that use of transvaginal mesh devices to treat stress urinary incontinence and pelvic organ prolapse has exposed women to avoidable harms. Part of the problem may lie with the governance of medical devices, which has enabled new devices to be brought to market with inadequate evidence, and over 100000 litigation cases are now under way. 1 We consider how improvements to both regulatory approvals and the structures supporting evidence based practice among clinicians could help prevent similar problems in the future.

\section{Clinical background}

Mesh has been used to treat stress urinary incontinence and prolapse for the past 20 years because it overcomes the need to harvest native tissue, is less invasive, takes less surgical time, and has been thought to reduce the risk of recurrent prolapse. Transvaginal mesh made from polypropylene is used to treat stress urinary incontinence. This operation involves creating a bladder sling using a strip of mesh (referred to as tape) that is inserted through vaginal and abdominal incisions. Similar techniques are used to treat pelvic organ prolapse, with vaginal mesh made from the same material used to support the tissues which hold the vaginal walls and uterus in place. Although the evidence for use of bladder slings is to some extent reassuring, 2 that for vaginal prolapse mesh has been controversial.3

The many different brands of mesh devices currently available are predominantly polypropylene based. However, they differ in pore size (macroporous or microporous) and fibre configuration (braided, woven, or monofilament); fibres can include a non-absorbable coat with a thin layer of porous collagen, and surface area can vary.4 Polypropylene mesh shrinks substantially after implantation, by up to $50 \%$ after four weeks, which may be associated with serious complications such as severe pain, dyspareunia, and tissue erosion, and often requires repeat surgical intervention.5

Bacterial contamination is another cause of problems and is found in up to a third of removed meshes. 6 Laboratory data show surface roughness can promote migration of bacteria along fibres, 7 and animal model data show that greater surface area (multifilament meshes have twice the surface area of monofilament meshes) enhances bacterial adherence, 8 which may further promote infection. Infected multifilament meshes have to be removed, 9 and colonisation can occur whatever intraoperative sterility procedure is used.10 Because meshes are designed to encourage ingrowth of nerves that accompany tissue and blood vessels (the aim of which is to reinforce weakened pelvic tissues), when complications occur mesh can be difficult to remove. 1112

The accumulating reports of harms attributed to mesh have resulted in more than 100000 women suing manufacturers globally. Over 1000 women have brought cases in the UK,1314 and more than 3000 women are reported to be in litigation in Canada.15 The number of US cases under litigation outweighs FDA spontaneous reports by 25 times (100 731 litigation cases v 3979 FDA reports), suggesting substantial under-reporting of harms to regulatory bodies. What went wrong? 


\section{Inadequate regulatory processes}

In the US, transvaginal meshes were initially class II devices (lower risk), permitting them to be cleared for marketing on the basis of equivalence to existing products (predicates) that are already are on the market (box 1).19 Over time, multiple submissions can lead to predicate creep, whereby the numerous changes result in a new device that is very different from the original predicate device.

\section{US and European device regulation}

In the US the Food and Drug Administration is responsible for all aspects of device regulation. Lower risk devices (class II) can be cleared for marketing through the less burdensome $510(\mathrm{k})$ review process. This requires the manufacture to show that the device is substantially equivalent to, or at least as safe and effective as a legally marketed device or "predicate." The mechanism allows the creation of a chain of equivalence, in some cases leading back to devices approved before 1976 (when the Medical Device Regulation Act [21 CFR 807.92] was introduced), 16 and before clinical evidence was required.17

In Europe approval of devices is managed through over 50 commercial organisations known as notified bodies. A manufacturer selects a notified body to certify a new device.18 The notified body asks the manufacturer for information about the device and assesses conformity to the essential requirements in three legal directives. If the notified body approves the application, it issues a certificate of conformity, which allows the manufacturer to market the product anywhere in Europe. Clinical data to show equivalence to previous devices can be as limited as a critical narrative review of the relevant scientific literature.

In our linked BMJ Open paper we traced marketing clearance for 61 mesh devices back through a chain of equivalence claims to only two unique originating devices approved in 1985 and 1996.17 We found no evidence of any new clinical trial data at the time of device approval for all of these 61 devices, with empirical evidence of effectiveness from randomised trials emerging on average five years after approval (range 1 to 14 years).

However, changes in design should have alerted regulators to important differences in the technological characteristics of the mesh that should have negated the use of equivalence.4 As an example, one of the early devices, the Protegen sling, which was made from polyester, continued to be used as a predicate for more modern devices even though they were made from polypropylene, and despite the Protegen sling being removed from the market.

\section{Reliance on animal testing for biocompatibility}

Before marketing, meshes are assessed to determine toxicity to cells (cytotoxicity) how irritative a product or compound is to skin (intracutaneous studies), and whether a material contains chemicals that cause adverse local or systemic effects after prolonged exposure (sensitisation studies). Cytotoxicity is generally examined using mouse media containing fibroblasts in culture, 
which are then examined for 48 hours for signs of reactivity. In the intracutaneous studies, which may use as few as two animals,20 observations for erythema are carried out for up to 72 hours after intracutaneous injections of appropriate extracts and controls. Such studies are unlikely to reflect the benefit-harm profile of implantable devices that remain in humans for decades.

However, arguably the most important test is measurement of how compatible a device is with a biological system, so called biocompatibility. The purpose is "protection of humans from potential biological risks arising from the use of medical devices." 21 Mice, rats, and rabbits are routinely used to evaluate biocompatibility because they are easy to handle and low cost and there is experience with using them. 20 However, it is uncertain how closely animal models predict human responses to implantable devices. When a device is to be implanted it is vital that studies should closely approximate intended clinical use to provide a reasonable assessment of potential harms.22

\section{Slow emergence of long term evidence}

In 2005, a Cochrane review of surgery for stress urinary incontinence or pelvic organ prolapse highlighted the need for studies with longer term follow-up.23 At the same time, the UK National Institute for Health and Care Excellence (NICE) stressed the need to inform women about the lack of long term outcomes data.24

The longer term evidence, however, identified serious concerns. In 2009, a review of 127 cases of transvaginal repair using synthetic mesh cited tissue erosion rates over $10 \%, 25$ and complications requiring reoperation were reported to be more common with vaginal mesh kits than with traditional vaginal surgery.26 A 2010 randomised trial of colpopexy versus prolapse repair with mesh was stopped early because of high erosion rates in the mesh group (16\% at three months).27 A 2011 systematic review of 110 studies of vaginal prolapse repair with graft materials materials (91 of which were non-absorbable synthetic mesh materials) reported an overall erosion rate of $10.3 \%$ (range $0 \%$ to $30 \%$ ). Dyspareunia was described in 70 studies and occurred at a rate of $9.1 \%$ (range $0 \%$ to $67 \%$ ). 28

In 2011, the FDA published a systematic review of vaginal mesh studies from 1996 to 2011 and reported some pressing problems, overturning its 2008 advice that mesh complications were rare.29 The FDA also used information reported to its Manufacturer and User Device Experience (MAUDE) database. The FDA cited 3979 reports of serious complications associated with urogynaecological surgical mesh products. The most common complications included erosion of vaginal mesh into surrounding tissues, followed by pain, infection, bleeding, dyspareunia, and organ perforation.29 Contrary to previous FDA statements, the review concluded that "while transvaginal [pelvic organ prolapse] repair with mesh often restores anatomy, it has not been shown to improve clinical benefit over traditional non-mesh repair."

In 2012, the FDA asked 33 manufacturers of surgical meshes to conduct 119 new safety studies.17 Our linked BMJ Open study shows that in response, the manufacturers instead 
ceased market distribution in 79 (66\%) cases and changed the indication in $26(22 \%)$ cases. In two orders the manufacturer reported it was no longer in business and one reported the device was not a mesh. In four orders the manufacturer requested their multiple orders should be consolidated into one leaving seven studies under way to assess the risks of harms.17 The FDA reclassified vaginal mesh for pelvic organ prolapse repair from a class II device to a class III device in 2016,30 requiring more stringent testing in trials before clearance.

In the UK the Medicines and Healthcare Products Regulatory Agency (MHRA) published an assessment of the clinical effectiveness of transvaginal meshes for stress urinary incontinence and pelvic organ prolapse in 2014, based on data from an overview of systematic reviews and reports of adverse events.31 The MHRA stated that the evidence from published reviews was insufficient to assess the benefit to harm balance of meshes for specific procedures; however, it noted differences in patient characteristics, surgical expertise and experience, and patient aftercare, which can affect susceptibility to adverse events.

Although the MHRA included only data from randomised trials in its literature review, it largely failed to report between group comparisons for mesh versus native tissue repair surgery and did not report the quality of the evidence for the outcomes assessed. Since 2005, the MHRA has received a total of 110 reports of adverse events with vaginal mesh implants for pelvic organ prolapse: the most common reports were pain (39), extrusion/erosion (65), infection (21), relapse of conditions/urinary symptoms (20), perforation (16), and dyspareunia (18). ). In contrast to the FDA, the MHRA concluded that the "benefits outweigh the risks" for use of mesh and in their review of the information available to them. They stated 'there appears to be no evidence that vaginal mesh implants are unsafe.31Table 1 compares the assessments of the two organisations.

In 2016, Cochrane updated its review of the surgical management of pelvic organ prolapse and reported higher reoperation rates for women treated with mesh than with native tissue. Evidence from 37 trials including 4023 women reported that, compared with native tissue repair, mesh probably reduced the awareness of prolapse and repeat surgery for prolapse. However, around $10 \%$ of women with mesh would require reoperation for prolapse, urinary incontinence, or mesh exposure.32

Although these various reviews have attempted to explore long term outcomes, they are hindered by methodological shortcomings, high drop-out rates, and sparse long term data. In a UK trial of transvaginal tape in 344 women, only 177 (51\%) provided data at five years, and full subjective and objective data were available for just 121 (35\%) women.33 Long term cohort studies have similarly reported high drop-out rates (median 40\% loss to follow-up).24 The FDA considers that mesh trials have been poorly designed and poorly conducted and have failed to account for variable lengths of patient follow-up.29

\section{Usage and response to evidence}


Annual numbers of UK NHS operations for stress urinary continence rose by $28 \%$ from its introduction in 1997-98 through to 2005-06. Similar trends have been observed for mesh use in prolapse. For example, use increased by $19 \%$ between 2003 and 2006.42

Vaginal mesh procedures also increased in the US. Numbers tripled between 2005 and 2010,43 despite emerging evidence highlighting the need for better data on long term outcomes24 and an FDA safety alert in October 2008. It was not until after the FDA ordered postmarketing studies to assess safety and effectiveness in January 2012 and evaluate risks in April 2014 that use of mesh for prolapse began to decrease in the US.29

\section{Changes to device regulation}

In recognition of the growing problems, many countries have already reclassified mesh as high risk, and NICE is recommending transvaginal mesh should not be used to treat vaginal prolapse because of safety concerns.34 New EU regulations, published in May 2017, mean that clinical investigations for class III and implantable medical devices will be required to provide evidence of safety and performance.35 Notified bodies will also be redesignated under the new regulations, and those assessing high risk medical devices will be scrutinised to ensure compliance with the regulations. Notified bodies will have to inform competent authorities of their high-risk device approvals, and the competent authority will be able, based on reasonable concerns, to request further advice from expert panels about safety and performance, an important change from the old regulations.

However, there is a three year transition period before these rules fully come into force, in May 2020. We think that these changes are insufficient, and the long delay in implementation does not represent a timely response to patients' needs. The FDA's decision in 2016 to make mesh a class III, high risk medical device means that in the US manufacturers already have to provide clinical trial evidence of the safety and effectiveness of their products before market approval.

In the case of vaginal mesh devices, evidence from large pragmatic trials did not emerge until 20 years after the first products were introduced3637 and 12 years after the call for longer term studies.2324 In our view, to be considered safe and approved for widespread use, long term implantable devices should have been evaluated in studies with follow-up of at least five years. Limited access could be provided through temporary licences that restrict use to within clinical trials with long follow-up. This would ensure that safety and effectiveness data were available before full marketing authorisation (referred to in the US as investigational device exemption).3839 Our proposed model for device development, which is based on methods used for drugs and the IDEAL-D framework, works towards this (table 2).

Finally, we recommend that a patient registry should be established for all implantable devices to enable long term follow-up and surveillance. Twenty years after the first use of vaginal mesh, device manufacturers, the FDA, and professional organisations established the pelvic floor disorders registry. Such registries should include unique device identification so that any 
shortcomings can be more readily tracked, patterns of use monitored, and patients later judged to be at risk more easily identified.

Key messages

- Thousands of women are suing manufacturers after developing complications from vaginal mesh devices

- Many of these devices were approved on the basis of equivalence to older approved devices despite important changes

- Evidence consistently pointed to a lack of long term data to inform use of vaginal mesh devices

- Regulation of implanted devices needs to recognise the higher potential risk

- Registries should be required for all implantable devices to track any problems

\section{Footnotes}

Contributors and sources: $\mathrm{CH}$ has extensive expertise in device regulation and has worked with patients, individuals and the media to prepare this article. $\mathrm{CH}$ and $\mathrm{BG}$ have an active interest in regulatory science, and all authors have experience and expertise in the assessment of adverse effects. For the preparation of this article we used FDA and MHRA documents, EU regulatory frameworks and research PubMed for relevant systematic review evidence $\mathrm{CH}$ wrote the first draft, IO, JKA, and $\mathrm{CH}$ collated the data and IO, BG, AP, JKA, and KRM all contributed to the data interpretation and development of the manuscript and approved the final draft. $\mathrm{CH}$ is the guarantor.

Competing interests: We have read and understood BMJ policy on declaration of interests and declare the following: $\mathrm{CH}$ has been an expert witness in a mesh medical device legal case, but took no payment. He has received financial remuneration from an asbestos legal case. He has received expenses in the past from the FDA to attend a working group on device regulation. JKA has published papers and edited textbooks on adverse drug reactions; he has also acted as an expert witness in cases related to adverse drug reactions. BG reports personal fees from intermittent additional personal income from speaking and writing for lay audiences on problems in science and medicine including regulatory shortcomings. $\mathrm{CH}$ receives funding from the National Institute for Health Research (NIHR) School of Primary Care. BG receives funding from the Laura and John Arnold Foundation.

Provenance and peer review: Not commissioned; externally peer reviewed.

\section{References}

1. McCarthy J. Hinch backs women victims of medical "scandal" photos, video. Newcastle Herald 2017 Feb 3.http://www.theherald.com.au/story/4443127/hinch-backswomenvictims-of-medical-scandalphotos-video/ 
2. KeltieKEIneilSMongaA. Complications following vaginal mesh procedures for stress urinary incontinence: an 8 year study of 92,246 women. Sci Rep2017;7:12015. doi:10.1038/s41598-017-11821-w28931856

3. ShahHNBadlaniGH. Mesh complications in female pelvic floor reconstructive surgery and their management: A systematic review. Indian J Urol2012;28:129-53. doi:10.4103/0970-1591.9845322919127

4. OstergardDR. Vaginal mesh grafts and the Food and Drug Administration. Int Urogynecol J2010;21:1181-3. doi:10.1007/s00192-010-1227-920683578

5. FeinerBMaherC. Vaginal mesh contraction: definition, clinical presentation, and management. Obstet Gynecol2010;115:325-30. doi:10.1097/AOG.0b013e3181cbca4d20093906

6. OstergardDR. Degradation, infection and heat effects on polypropylene mesh for pelvic implantation: what was known and when it was known. Int Urogynecol J2011;22:771-4. doi:10.1007/s00192-011-1399-y21512830

7. CoughlinRWMullenDBrancieriMRezmanVViethRF. Surface roughness enhances upward migration of bacteria on polymer fibers above liquid cultures. J Biomater Sci Polym Ed1999;10:827-44. doi:10.1163/156856299X0090010487317

8. KlingeUJungeKSpellerbergBPirothCKlosterhalfenBSchumpelickV. Do multifilament alloplastic meshes increase the infection rate? Analysis of the polymeric surface, the bacteria adherence, and the in vivo consequences in a rat model. J Biomed Mater Res2002;63:765-71. doi:10.1002/jbm.1044912418022

9. GoldsteinHS. Selecting the right mesh. Hernia1999;3:23-6doi:10.1007/BF01576737.

10. VollebregtATroelstraAvan der VaartCH. Bacterial colonisation of collagen-coated polypropylene vaginal mesh: are additional intraoperative sterility procedures useful? Int Urogynecol J Pelvic Floor Dysfunct2009;20:1345-51. doi:10.1007/s00192-009-0951-519727538

11. OstergardDR. Degradation, infection and heat effects on polypropylene mesh for pelvic implantation: what was known and when it was known. Int Urogynecol J2011;22:771-4. doi:10.1007/s00192-011-1399-y21512830

12. BarskiDDengDY. Management of mesh complications after SUI and POP repair: review and analysis of the current literature. Biomed Res Int2015;2015:831285. doi:10.1155/2015/83128525973425

13. Scottish Government. The Scottish independent review of the use, safety and efficacy of transvaginal mesh implants in the treatment of stress urinary incontinence and pelvic organ prolapse in women: final report. 2017. http://www.gov.scot/Publications/2017/03/ 3336/9

14. Sanghani R. Why hundreds of women are suing the NHS over vaginal mesh surgery. Telegraph 2017 Apr 18. http://www.telegraph.co.uk/health-fitness/body/hundredswomensuing-nhs-vaginal-mesh-surgery-felt-like-had/

15. Lawsuits being settled in surgical mesh complications. CTVNews 2015 Jan 13. http:// www.ctvnews.ca/health/lawsuits-being-settled-in-surgical-mesh-complications-1.21874

16. FDA. Code of federal regulations title 21. https://www.accessdata.fda.gov/scripts/cdrh/ cfdocs/cfcfr/CFRSearch.cfm?CFRPart=807 
17. HeneghanC GoldacreB Onakpoyal. Trials of transvaginal mesh devices for pelvic organ prolapse: a systematic database review of the US FDA approval process. BMJ Open2017;7:e017125. doi:10.1136/bmjopen-2017-017125.

18. MHRA. Notified bodies for medical devices. 2017. https://www.gov.uk/government/ publications/notified-bodies-for-medical-devices/notified-bodies-for-medical-devices (accessed 30 Oct 2017).

19. SorensonCDrummondM. Improving medical device regulation: the United States and Europe in perspective. Milbank Q2014;92:114-50. doi:10.1111/14680009.1204324597558

20. Biocompatibility safety assessment of medical devices: FDA/ISO and Japanese guidelines

(page 8). http://www.toxikon.com/userfiles/files/

BiocompatibilityAssessmentFDAJapaneseGuideline.pdf

21. ISO. Biological evaluation of medical devices-Part 1: Evaluation and testing within a risk

management process. https://www.iso.org/obp/ui/\#iso:std:iso:10993:-1:dis:ed-5:v1:en

22. Pacific BioLabs. Assessing biocompatibility: biological test methods. http://www. pacificbiolabs.com/bio_methods.asp.

23. BezerraCABruschiniHCodyDJ. Traditional suburethral sling operations for urinary incontinence in women. Cochrane Database Syst Rev2005;3:CD001754.16034866

24. National Collaborating Centre for Women's and Children's Health. Urinary incontinence: the management of urinary incontinence in women.RCOG Press, 2011.

25. GanjFAlbeanuOABedestaniANolanTEChessonRR. Complications of transvaginal monofilament polypropylene mesh in pelvic organ prolapse repair. Int Urogynecol J Pelvic

Floor Dysfunct2009;20:919-25. doi:10.1007/s00192-009-0879-919582383

26. DiwadkarGBBarberMDFeinerBMaherCJelovsekJE. Complication and reoperation rates after apical vaginal prolapse surgical repair: a systematic review. Obstet Gynecol2009;113:367-73. doi:10.1097/AOG.0b013e318195888d19155908

27. IglesiaCBSokolAISokolER. Vaginal mesh for prolapse: a randomized controlled trial. Obstet Gynecol2010;116:293-303. doi:10.1097/AOG.0b013e3181e7d7f820664388

28. AbedHRahnDDLowensteinLBalkEMClemonsJLRogersRGSystematic Review Group of the Society of Gynecologic Surgeons. Incidence and management of graft erosion, wound granulation, and dyspareunia following vaginal prolapse repair with graft materials: a systematic review. Int Urogynecol J2011;22:789-98.

doi:10.1007/s00192-011-1384-521424785

29. FDA. Urogynecologic surgical mesh: update on the safety and effectiveness of transvaginal placement for pelvic organ prolapse. 2011. www.fda.gov/downloads/medicaldevices/ safety/alertsandnotices/UCM262760.pdf

30. Food and Drug Administration, HHS. Obstetrical and gynecological devices. Reclassification of surgical mesh for transvaginal pelvic organ prolapse repair; final 
order.

Fed Regist2016;81:353-61.26742182

31. UK Medicines and Healthcare Products Regulatory Agency. A summary of the evidence on the benefits and risks of vaginal mesh implants. 2014.

https://www.gov.uk/government/

uploads/system/uploads/attachment_data/file/402162/Summary_of_the_evidence_on_ The_benefits_and_risks_of_vaginal_mesh_implants.pdf

32. MaherCFeinerBBaesslerKChristmann-SchmidCHayaNMarjoribanksJ. Transvaginal mesh or grafts compared with native tissue repair for vaginal prolapse. Cochrane Database

Syst Rev2016;2:CD012079.26858090

33. WardKLHiltonPUK and Ireland TVT Trial Group. Tension-free vaginal tape versus colposuspension for primary urodynamic stress incontinence: 5-year follow up. BJOG2008;115:226-33. doi:10.1111/j.1471-0528.2007.01548.x17970791

34. WiseJ. NICE to ban mesh for vaginal wall prolapse. BMJ2017;359:j5523

35. Salvatore V. Tightening medical device safety. PM Live 2017 Sep 11.http://www.pmlive. com/pharma_intelligence/tightening_medical_device_safety_1200683

36. FaircloughEMyersJSmithARBreemanSReidF. A UK questionnaire survey of current techniques used to perform pelvic organ prolapse repair. Int Urogynecol J2017; [Epub ahead of print]. doi:10.1007/s00192-017-3273-z28150029

37. GlazenerCMBreemanSEldersAPROSPECT Study Group. Mesh, graft, or standard repair for women having primary transvaginal anterior or posterior compartment prolapse surgery:

two parallel-group, multicentre, randomised, controlled trials (PROSPECT). Lancet2017;389:381-92. doi:10.1016/S0140-6736(16)31596-328010989

38. FDA. Device advice: investigational device exemption (IDE). https://www.fda.gov/ medicaldevices/deviceregulationandguidance/howtomarketyourdevice/ investigationaldeviceexemptionide/

39. MHRA. Guidance. Notify MHRA about a clinical investigation for a medical device. 2017. https://www.gov.uk/guidance/notify-mhra-about-a-clinical-investigation-for-amedicaldevice

40. BarkunJSAronsonJKFeldmanLSBalliol Collaboration. Evaluation and stages of surgical innovations. Lancet2009;374:1089-96. doi:10.1016/S0140-6736(09)61083-719782874

41. SedrakyanACampbellBMerinoJGKuntzRHirstAMcCullochP. IDEAL-D: a rational framework

for evaluating and regulating the use of medical devices. BMJ2016;353:i2372. doi:10.1136/bmj.i237227283585

42. HiltonP. Long-term follow-up studies in pelvic floor dysfunction: the Holy Grail or a realistic aim?BJOG2008;115:135-43. doi:10.1111/j.1471-0528.2007.01557.x17999691

43. Jonsson FunkMEdenfieldALPateVViscoAGWeidnerACWuJM. Trends in use of surgical mesh for pelvic organ prolapse. Am J Obstet Gynecol2013;208:79.e1-7. doi:10.1016/j.ajog.2012.11.00823159692 